\title{
Resolution, data quality metrics and diffraction limits: consolidation and outlook for macromolecular crystallography.
}

\author{
Vonrhein, C. ${ }^{a}$, Bricogne, Ga . Tickle, I.J. ${ }^{a}$, Flensburg, C. ${ }^{a}$, Fogh, R. ${ }^{a}$, Keller, P. ${ }^{a}$, Paciorek, W. ${ }^{a}$, \\ Sharff, A. $^{\mathrm{a}}$ \\ a Global Phasing Ltd, Cambridge CB3 0AX, UK (proc-develop@globalphasing.com).
}

The assumption that the diffraction limit of a macromolecular X-Ray diffraction dataset can be equated with the notion of resolution (in the optical sense, i.e. to describing its ability to resolve features), and therefore with the final model quality that can be achieved by refinement against it, is being revisited. New approaches for fully describing the three-dimensional characteristics of data collected from real-life crystals on current detectors, beamlines and instruments have fuelled a flurry of activity around the general theme of anisotropy over the past three years $[1,2,3]$.

Consolidating and extending the currently available data quality metrics to accommodate this evolution is a major undertaking. It involves re-assessing numerous preconceptions present in the existing literature [4], in mmCIF deposition dictionaries and in program documentations, as well as the current systems for (i) reporting those quality indicators to users of software and beamlines, (ii) enabling the full handling of all relevant data during deposition, and (iii) providing guidance for authors and readers of publications. For this purpose a workshop was recently organised by Global Phasing Ltd. and held at the ESRF (Grenoble) on April 9-11, 2019. It gathered a total of 30 invited participants encompassing developers of primary processing programs, auto-processing pipeline developers from multiple synchrotrons, high-volume users of MX data collection facilities, as well as diffraction database (viz. ISPyB) specialists and a wwPDB representative [5].

Here we will present an overview of the current state-of-the art, of how the necessary changes could be accommodated in existing systems of reporting/archiving (e.g. [6]), and of how the MX community as a whole might want to respond to the need to improve on its current practices.

[1] Tickle, I.J. et al (2016). STARANISO. Global Phasing Ltd., Cambridge, UK.

[2] http://staraniso.globalphasing.org/

[3] http://staraniso.globalphasing.org/cgi-bin/PDBpeep.cgi

[4] Rupp, B. (2018). Structure 26, 919-923. (doi:10.1016/j.str.2018.04.013)

[5] https://www.globalphasing.com/DQMW/

[6] Vonrhein, C. et al (2011). Acta Cryst. D67, 293-302. (doi:10.1107/S0907444911007773) 